\title{
Segmental Hyperhidrosis as a Manifestation of Spinal and Paraspinal Disease
}

\author{
Valerie Schulz, Donna Ward and Dwight E. Moulin
}

\begin{abstract}
Background: Segmental hyperhidrosis is an uncommon finding which is usually associated with irritation or infiltration of pre-ganglionic sympathetic fibres or the sympathetic chain. Methods: We report two cases of segmental hyperhidrosis with striking clinical features. Results: In one case, a mesothelioma produced ipsilateral simultaneous underactivity and overactivity of sympathetic outflow and in the other case a thoracic central disc herniation was probably responsible for a band of sweating which clearly extended beyond the segmental level of injury. Conclusion: Segmental hyperhidrosis should trigger a search for structural disease in the spinal and paraspinal region.
\end{abstract}

\begin{abstract}
RÉSUMÉ: Hyperhydrose segmentaire comme manifestation d'une maladie spinale et paraspinale. Introduction: L'hyperhydrose segmentaire est une observation rare qui est habituellement associée à une irritation ou à une infiltration des fibres sympathiques pré-ganglionnaires ou de la chaîne sympathique. Méthodes: Nous rapportons deux cas d'hyperhydrose segmentaire accompagnée de manifestations cliniques inusitées. Résultats: Dans un cas, un mésothéliome a provoqué simultanément une hypoactivité et une hyperactivité sympathique ipsilatérale et dans l'autre cas, une hernie discale centrale au niveau thoracique était probablement responsable d'une bande de transpiration qui s'étendait au-delà du niveau segmentaire de la lésion. Conclusion: Dans l'hyperhydrose segmentaire, on doit rechercher une maladie structurale dans la région spinale et paraspinale.
\end{abstract}

Can. J. Neurol. Sci. 1998; 25: 325-327

Unilateral hyperhidrosis is an uncommon finding which has been reported in a variety of clinical conditions including hypothalamic tumours, spinal cord injury and cerebral infarction. ${ }^{1-3}$ Segmental hyperhidrosis can be unilateral or bilateral and is usually associated with a structural lesion causing irritation or infiltration of pre-ganglionic sympathetic fibres or the sympathetic chain. ${ }^{4}$

We report two cases of segmental hyperhidrosis with striking clinical features. In one case there was simultaneous evidence of underactivity and overactivity of the sympathetic nervous system due to the same disease process and in the other the band of sweating clearly extended beyond the segmental level of injury.

\section{Patient 1}

A 49-year-old electrician with a long history of asbestos exposure was admitted to hospital in June 1993 for investigation and treatment of progressive shortness of breath. Chest $x$-ray showed an extensive left pleural-based mass. He underwent a thoracotomy and subtotal resection of the tumour through the 5th intercostal space which yielded a diagnosis of mesothelioma. Following surgery he developed aching pain and numbness along the incision line and over the following year experienced a $30 \mathrm{~kg}$ weight loss, increasing shortness of breath and escalating pain involving the left upper back, chest wall and abdomen. He described a heavy "crampy" pain which was most marked along the medial border of the scapula and around the chest wall just below the thoracotomy incision. In August 1994 he became aware of a band of sustained increased sweating just below the thoracotomy incision and in October he developed a partial droop of the left upper lid. His pain was managed with escalating doses of oral hydromorphone and by October 1994 he had reached a dose of $24 \mathrm{mg} \mathrm{q} 4 \mathrm{~h}$.

Examination revealed an alert, cachectic gentleman with a thoracic scoliosis concave to the left. He had a Horner's syndrome with decreased sweating involving the left face and upper extremity. Motor examination showed mild symmetrical weakness involving the shoulder and hip girdles. There was blunting to pinprick involving the left chest wall in T5-T9 distribution without hyperpathia. There was hyperhidrosis and pallor of the skin in the same distribution, which was more marked posteriorly. The pattern of sweating was easily delineated with the Quinizarin Sweat Test ${ }^{5}$ (Figure 1). Quinizarin powder turns dark brown to purple in the presence of sweat. CT scan of the chest revealed tumour encasing the left lung with extensive paraspinal disease extending from T1-T10 (Figure 2). MRI scan of the thoracic spine showed no evidence of epidural disease.

The patient was started on a continuous subcutaneous hydromorphone infusion to provide adequate pain control. He received radiation therapy to the left chest wall and paraspinal region without any further benefit except for some diminution in hyperhidrosis. He died in December 1994.

\section{Patient 2}

A previously well 49 -year-old plant supervisor developed bilateral flank pain in April 1995 without any obvious precipitating event. He described constant "squeezing, tightening" pain with intermittent radiation around the trunk at the costal margins. The pain was worse on deep inspiration and with prolonged sitting and forced him to switch to modi-

From the Departments of Anesthesia (V.S.) and Clinical Neurological Sciences (D.E.M.), University of Western Ontario, London, Ontario and Department of Family Medicine (D.W.), Grand River Hospital, Kitchener, Ontario.

RECEIVED NOVEMBER 6, 1997. ACCEPTED IN FINAL FORM APRIL 21, 1998.

Reprint requests to: D. Moulin, Department of Clinical Neurological Sciences, London Health Sciences Centre, Victoria Campus, 375 South Street, London, Ontario, Canada N6A 4G5 


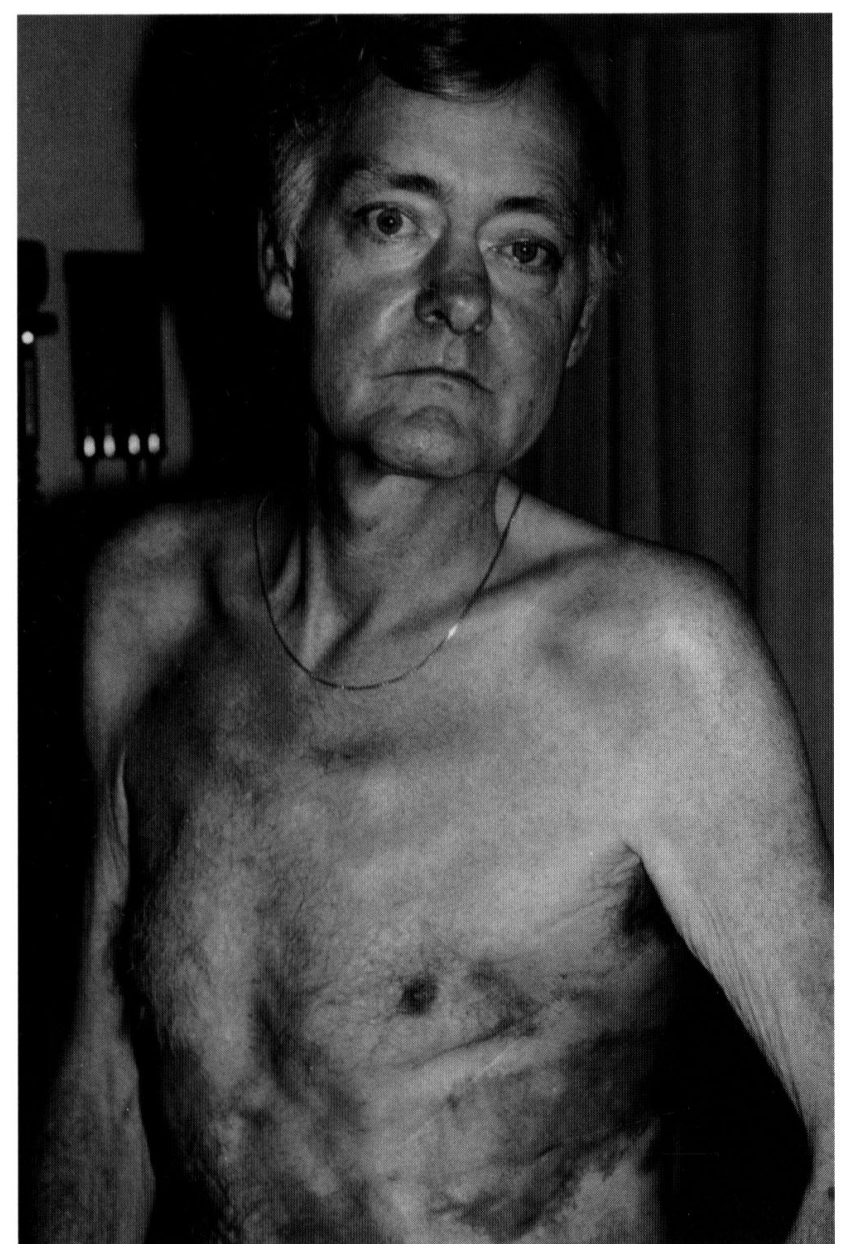

Figure 1: Patient 1 with extensive mesothelioma involving the left lung. Figure shows left Horner's syndrome and hyperhidrosis of the left chest wall in T5-T9 distribution following application of quinizarin powder.

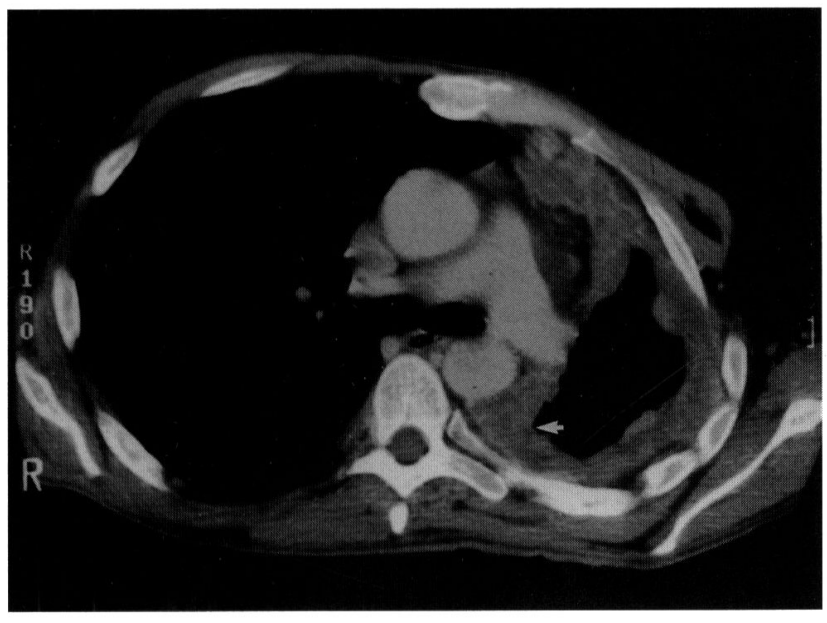

Figure 2: CT scan of chest at T7.T8 in Patient 1 showing tumour encasing left lung with prominent paraspinal disease (arrow). fied work duties. There was no history of leg weakness, unsteadiness or sphincter disturbance and he remained systemically well.

Examination in October 1995 revealed a well nourished gentleman who was not tender over the thoracic spine or in either flank. Motor examination revealed normal bulk, tone and power. The deep tendon reflexes were symmetrical and the plantar responses were flexor. There was bilateral blunting to pinprick in segmental distribution at T7-T8 without allodynia or hyperalgesia. Vibration sense and position sense were intact. There was a distinct band of bilateral hyperhidrosis extending from T6-T10. MRI scan of the thoracic spine showed a moderate central disc bulge at T7-T8 with indentation of the thecal sac but no obvious nerve root impingement (Figure 3 ). In consultation with the Orthopedic Service, the decision was made to provide conservative management. He was intolerant to low dose amitriptyline and did not respond to transcutaneous electrical nerve stimulation. Acetaminophen with codeine $30 \mathrm{mg}$ provided partial relief and he was able to get back to work full time in April 1996. Telephone follow-up in January 1997 revealed that he continued to require acetaminophen with codeine to provide adequate pain control and that his hyperhidrosis persisted unchanged.

\section{DisCuSSION}

Peripheral nerve injury may simply produce a sensory and autonomic deficit in the territory of the involved nerve. However, nerve injury can also produce positive symptoms such as pain and hyperhidrosis. The former is common and the latter is rare as illustrated by the report of Hepper et al. ${ }^{6}$ where $92 \%$ of 94 patients with thoracic inlet (Pancoast) tumours had pain as the initial symptom but only $2 \%$ developed hyperhidrosis of the upper extremity. Despite this discrepancy in the prevalence of symptomatology, there are likely similarities in the pathophysiology of neuropathic pain and hyperhidrosis. Neuropathic pain can

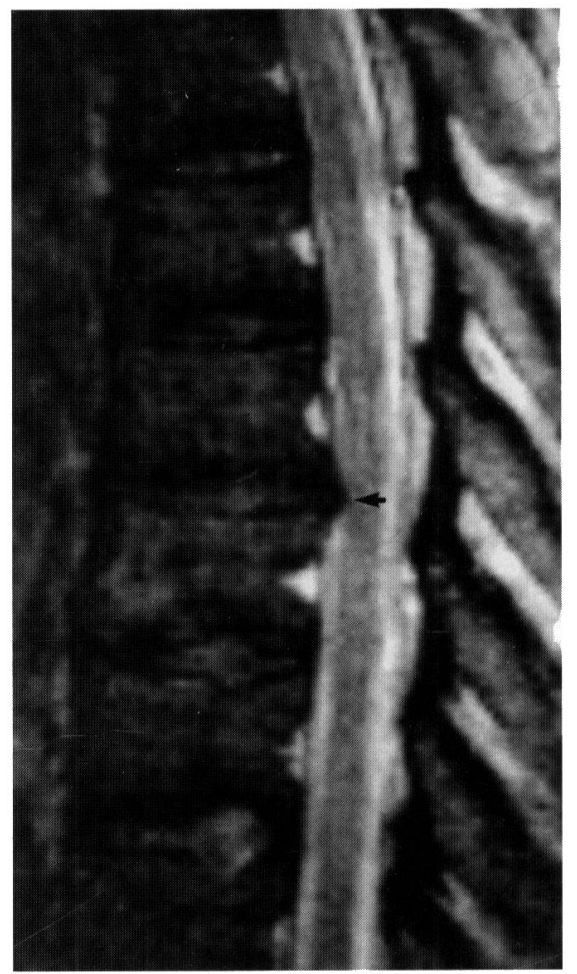

Figure 3: T2-weighted sagittal MRI scan of thoracic spine in Patient 2 showing moderate central disc bulge at T7-T8 with indentation of the thecal sac (arrow). 
be generated by ectopic discharge in dorsal root ganglia cells and in local patches of demyelination along the axon. ${ }^{7,8}$ Similar ectopic activity in sympathetic ganglia and gray rami communicantes may result in hyperhidrosis. A constriction injury to the sciatic nerve of the adult rat can produce sympathetic sprouting in the dorsal root ganglia within four days of the insult. ${ }^{9}$ Injured nerves are also capable of cross-excitation or ephaptic transmission. ${ }^{10}$ Accentuation of burning pain and hyperhidrosis by rubbing the skin in the affected area has been reported in a patient with a paravertebral malignancy. ${ }^{4}$ Finally, nerve transection can result in denervation supersensitivity of the dorsal horn ${ }^{11}$ and similar injury to pre-ganglionic sympathetic fibres may produce sustained discharges in sympathetic ganglia.

All of the above mechanisms could account for the unilateral segmental hyperhidrosis seen in Patient 1 . There are several reports of unilateral hyperhidrosis in association with intrathoracic malignancies. ${ }^{12-16} \mathrm{~A}$ common observation has been the gradual loss of hyperhidrosis and, in fact, the development of anhidrosis and a Horner's syndrome as a result of sympathetic paralysis - presumably due to progression from sympathetic irritation to sympathetic destruction. ${ }^{13,14}$ This is the first report of ipsilateral simultaneous underactivity and overactivity of sympathetic outflow which implies variable rates of tumour progression at different levels of the neural axis.

Hyperhidrosis contralateral to the major site of disease has also been described in patients with paravertebral plasmacytoma ${ }^{17}$ and metastatic adenocarcinoma. ${ }^{18}$ In another patient, a teratoma involving the psoas muscle was responsible for ipsilateral lower extremity anhidrosis and contralateral hyperhidrosis. ${ }^{19}$ These case reports clearly show that a tumour mass involving primarily one paravertebral gutter may produce ipsilateral destruction and contralateral excitation of the sympathetic chain. The Harlequin syndrome is characterized by unilateral facial flushing and sweating induced by heat in patients with a sympathetic defect on the opposite side. ${ }^{20}$

Hyperhidrosis may also be the product of non-malignant disease involving sympathetic fibres and is more likely to be reversible. Telford ${ }^{21}$ described two patients who had a cervical rib and hyperhidrosis of the ipsilateral hand. Following surgical resection, their symptoms rapidly resolved. Alternating Horner's syndrome and hyperhidrosis involving the face and upper chest have been described in a patient with cervical spinal cord injury. ${ }^{2}$ Following removal of dural adhesions, the hyperhidrosis resolved. Pool $^{22}$ described a patient with pain and hyperhidrosis of the left side of the trunk in T2-T12 distribution. The offending lesion was an osteoma that partially occluded the left T10-T11 intervertebral foramen. Hemi-laminectomy resulted in resolution of the hyperhidrosis and pain. Although Patient 2 did not have obvious nerve root impingement on the MRI scan, the local pain radiating around the costal margins, the sensory loss along T7-T8 and the striking segmental hyperhidrosis extending from T6-T10 were likely due to the central disc bulge at T7-T8 with secondary nerve root irritation. These observations of hyperhidrosis extending beyond the segmental level of injury can be explained by the anatomy of the sympathetic nervous system. Preganglionic fibres arising from a specific spinal segment project not only to the corresponding paravertebral ganglion but travel up and down the sympathetic trunk to synapse in multiple paravertebral ganglia. ${ }^{23}$ This is further supported by the observation that electrical stimulation of a single anterior root can result in widespread sympathetically-mediated effects over the distribution of five or more sympathetic ganglia. ${ }^{22}$

Although rare, segmental hyperhidrosis usually signals irritation or infiltration of nerve roots or the sympathetic chain and should trigger a search for structural disease in the spinal and paraspinal region.

\section{REFERENCES}

1. Poh SC. Bronchial carcinoma with hemilateral hyperhidrosis. Singapore Med J 1978; 19: 59-60.

2. Ottomo M, Heimburger RF. Alternating Horner's syndrome and hyperhidrosis due to dural adhesions following cervical spinal cord injury. J Neurosurg 1980; 53: 97-100.

3. Beum-Saeng Kim, Yeong-In Kim, Kwang-Soo Lee. Contralateral hyperhidrosis after cerebral infarction. Stroke 1995; 26(5): 896899.

4. Walsh JC, Low PA, Allsop JL. Localized sympathetic overactivity: an uncommon complication of lung cancer. J Neurol Neurosurg Psychiatry, 1976; 39: 93-95.

5. Baker AB, Baker LH eds. Clinical Neurology, Vol. 4. Harper \& Rowe Publishers, Philadelphia, PA, 1984.

6. Hepper NG, Herskovic T, Witten DM, et al. Thoracic inlet tumors. Ann Intern Med 1966; 64: 979-989.

7. Nordin M, Nystrom B, Wallin U, et al. Ectopic sensory discharges and paresthesias in patients with disorders of peripheral nerves, dorsal roots, and dorsal columns. Pain 1984; 20: 231-245.

8. Devor M. The pathophysiology of damaged peripheral nerves. Int: Wall PD, Melzack R, eds. Textbook of Pain. 2nd ed. London: Churchill-Livingston, 1989: 63-81.

9. Ramer MS, Bisby MA. Rapid sprouting of sympathetic axons in dorsal root ganglia of rats with a chronic constriction injury. Pain 1997; 70: 237-244.

10. Devor M, Wall PD. Cross-excitation in dorsal root ganglia of nerve injured and intact rats. J Neurophysiol 1990; 64: 1733-1746.

11. Loesser JD, Ward AA, White LE. Chronic deafferentation of human spinal cord neurons. J Neurosurg 1968; 29: 48-50.

12. Lindsay DC, Freeman JG, Record CO. Unilateral hyperhidrosis associated with underlying intrathoracic neoplasia. Thorax 1986; 41: 814-815.

13. Middleton WG. Bronchial carcinoma with pleural spread causing unilateral thoracic hyperhidrosis. Br Med J 1976; 2: 563.

14. Jegarajah S, Coutts II. Localized sympathetic overactivity: an unusual complication of bronchogenic carcinoma. $\mathrm{Br} \mathrm{J}$ Dis Chest 1977; 71: 300-302.

15. McEvoy M, Ryan E, Neale G, et al. Unilateral hyperhidrosis: an unusual presentation of bronchial carcinoma. Ir J Med Sci 1982; 151: 51-52.

16. Pleet DL, Mandel S, Neilan B. Paroxysmal unilateral hyperhidrosis and malignant mesothelioma. Arch Neurol 1983; 40: 256.

17. Lambert M, Kanyinda JM, Richard F, et al. Unilateral hyperhidrosis associated with intrathoracic IgD lambda myelomatous tumour. Clin Oncol 1993; 5: 65-66.

18. McCoy BP. Apical pulmonary adenocarcinoma with contralateral hyperhidrosis. Arch Dermatol 1981; 117: 659-661.

19. Brown RC. Unilateral lumbar sympathectomy due to retroperitoneal tumour. Br Med J 1978; 1: 1101.

20. Lance JW, Drummond PD, Gandevia SC, et al. Harlequin syndrome: the sudden onset of unilateral flushing and sweating. $J$ Neurol Neurosurg Psychiatry 1988; 51: 635-642.

21. Telford ED. Cervical rib and hyperhidrosis. Br Med J 1942; 2: 96.

22. Pool JL. Unilateral thoracic hyperhidrosis caused by osteoma of the tenth dorsal vertebra. J Neurosurg 1956; 13: 111-115.

23. Adams RD, Victor M, Ropper AH. Disorders of the autonomic nervous system and respiration. Principles of Neurology, 6th ed. New York: McGraw-Hill, 1997; 522-553. 